\title{
Análisis de la frecuencia de experimentación y consumo de drogas de alumnos de escuelas de nivel medio
}

\author{
Maria Soledad Burrone ${ }^{1}$ \\ Sonia Maria Villela Bueno² \\ Moacyr Lobo de Costa Jr ${ }^{3}$ \\ Julio Enders ${ }^{4}$ \\ Ruth Alicia Fernández ${ }^{5}$ \\ Gabriela Pereira Vasters ${ }^{6}$
}

En el presente trabajo se analiza la frecuencia de experimentación y consumo de drogas, en alumnos de escuelas de nivel medio de Córdoba, Argentina, en relación a variables sociodemográficas. De carácter analítico, la investigación se basó en los registros de la Secretaría de Programación para la Prevención de la Drogadicción y la Lucha contra el Narcotráfico. El procesamiento estadístico fue análisis bivariado, razón de riesgo e intervalos de confianza para cada variable. El consumo de alcohol fue más frecuente en los hombres, el $12,2 \%$ de los adolescentes probó algún tipo de droga ilícita, y los tranquilizantes son los psicofármacos más utilizados. El valor del consumo de drogas aumenta según aumenta el rango de edad, año de estudio y disponibilidad de dinero. Los hallazgos del presente trabajo identificaron una realidad epidemiológica que permitirá elaborar estrategias de prevención de adicción en los adolescentes.

Descriptores: Trastornos Relacionados con Sustancias; Adolescente; Epidemiología; Enfermería.

\footnotetext{
${ }^{1}$ Médica, Becaria, Escuela de Salud Pública, Facultad de Ciencias Médicas, Universidad Nacional de Córdoba, Argentina. E-mail: msburrone@gmail.com.ar.

2 Pedagoga, Doutor em Educação, Profesor Asociado, Escola de Enfermagem de Ribeirão Preto, Universidade de São Paulo, Centro Colaborador de la OMS para el Desarrollo de la Investigación en Enfermería, SP, Brasil. E-mail: smvbueno@eerp.usp.br.

${ }^{3}$ Estadístico, Doctor em Salud Publica, Profesor Asociado, Escola de Enfermagem de Ribeirão Preto, Universidade de São Paulo, Centro Colaborador de la OMS para el Desarrollo de la Investigación en Enfermería, SP, Brasil. E-mail: mlobojr@eerp.usp.br.

${ }^{4}$ Profesor Doctor, Facultad de Ciencias Médicas, Universidad Nacional de Córdoba, Argentina. E-mail: jeenders@gmail.com.

${ }^{5}$ Profesor Doctor, Facultad de Ciencias Médicas, Universidad Nacional de Córdoba, Argentina. E-mail: rfernandez@fcm.unc.edu.ar.

${ }^{6}$ Estudiante de Doctorado, Escola de Enfermagem de Ribeirão Preto, Universidade de São Paulo, Centro Colaborador de la OMS para el Desarrollo de la Investigación en Enfermería, Brasil. E-mail: gabi_vastesr@yahoo.com.br.
}

Correspondencia:

Sonia Maria Villela Bueno

Universidade de São Paulo. Escola de Enfermagem de Ribeirão Preto

Av. Bandeirantes, 3900

Bairro Monte Alegre

CEP: 14040-902 Ribeirão Preto, SP, Brasil

E-mail: smvbueno@eerp.usp.br 


\section{Análise da frequência de experimentação e consumo de drogas em alunos de escolas de Ensino Médio}

Este estudo teve como objetivo analisar a frequência de experimentação e consumo de drogas em alunos de escolas médias de Córdoba, Argentina, relacionada às variáveis sociodemográficas. Trata-se de estudo observacional e analítico, baseado no cadastro da Secretaria de Programação para a Prevenção da Drogadição e Luta contra o Narcotráfico. O processamento estatístico foi de análise bivariada, razão de risco e intervalos de confiança para cada variável. O consumo de álcool foi mais frequente entre os rapazes, $12,2 \%$ dos adolescentes experimentaram algum tipo de droga ilegal. Os tranquilizantes são os psicofármacos mais empregados. O valor do consumo de drogas cresce conforme o aumento da faixa etária, o nível de estudos e a disponibilidade de dinheiro. As descobertas do presente trabalho identificaram realidade epidemiológica útil para desenvolver estratégias de prevenção da adição em adolescentes.

Descritores: Transtornos Relacionados ao uso de Substâncias; Adolescente; Epidemiologia; Enfermagem.

\section{Analysis of the Frequency of Experimentation with and Consumption of Drugs in High-School Students}

The aim of this study was to survey the frequency of experimentation and consumption of drugs in high-school students in Cordoba, Argentina, relating it to socio-demographic variables. An observational-analytical method was employed, on the basis of the records of the Secretary for the Planning of Drug-Addiction Prevention and Fighting of DrugTrafficking. Statistical. Bivariate analysis, odds ratio and confidence interval were applied in the processing of each variable. Alcohol consumption was found to be more frequent among males. Of the subjects $12.2 \%$ had tried some type of illicit substance. Tranquilizers were the most commonly used psycho-medicine. Drug consumption increased with age, years of schooling and availability of money. Our detection of an epidemiological situation is deemed useful for prevention strategies for drug addiction among teenagers.

Descriptors: Substance-Related Disorders; Adolescent; Epidemiology; Nursing.

\section{Introducción}

Una de las metas de las instituciones dedicadas al abuso de drogas, es ayudar al público a comprender las causas del abuso y evitar su comienzo(1). El abuso de drogas tiene consecuencias serias en los hogares, las escuelas y en las comunidades. El consumo de cualquier droga ilícita o el uso inapropiado de drogas lícitas se considera abuso de drogas ${ }^{(2)}$. En este contexto, el consumo de drogas en los adolescentes constituye uno de los problemas prioritarios que enfrentan los administradores de salud pública en la mayoría de los países $^{(3)}$.

Así, desde la década del 90, se han identificado intervenciones eficaces con poblaciones más jóvenes para ayudar a prevenir las conductas de riesgo antes de que ocurra el abuso de drogas ${ }^{(4)}$.

La Organización de las Naciones Unidas estima una prevalencia mundial de $4,2 \%$ para cualquier droga ilícita en mayores de 15 años ${ }^{(5)}$, valor que para la Argentina alcanza el 2,3\%. La Primer Encuesta Nacional en Estudiantes de Enseñanza Media(6) realizada por la Secretaría de Programación para la Prevención de la Drogadicción y la Lucha contra el Narcotráfico (SEDRONAR) revela que más de la mitad de los adolescentes consumieron bebidas alcohólicas; esta droga junto al tabaco comparte la iniciación temprana. En los últimos 30 años se realizaron esfuerzos en la 
prevención de abuso de drogas y de los problemas asociados, siendo muy amplia la variedad de actuaciones propuestas $^{(7)}$. Las investigaciones realizadas sobre los programas de prevención nos permiten concluir que, en general, cuando el criterio de evaluación empleado es el consumo, los resultados positivos se limitan a aquellos programas que centran la intervención en la promoción de las competencias y en las variables psicosociales relacionadas con el consumo(8-10). A pesar de las campañas y programas preventivos realizados en los países desarrollados, entre un tercio y la mitad de los escolares experimentaron el tabaco antes de terminar la escolarización secundaria(3).

El tabaquismo, el alcoholismo y la adicción a otras drogas en el ser humano son resultado de una gran variedad de factores causales, donde la influencia del contexto ambiental y la repercusión en el ámbito social ha sido descrita ampliamente a nivel mundial, convirtiéndose en un problema de gran dimensión en la salud pública e internacional por las consecuencias que ocasiona en el individuo consumidor, su familia y comunidad.

En el informe del resumen estadístico sobre drogas de la Comisión Interamericana para el Control del Abuso de Drogas (CICAD), del año 2001, se informa que los datos podrían ser utilizados para establecer prioridades y desarrollar métodos para la ejecución de los programas. Bajo esta perspectiva la importancia de la presente investigación se basa en identificar las características de los alumnos de las escuelas de nivel medio, de la provincia de Córdoba, respecto las variables sociodemográficas. Estudios estadísticos de este tipo son de utilidad para implementar estrategias de prevención de la adicción en estos jóvenes y para la realización de nuevos estudios que evalúen el impacto de las intervenciones en este campo.

\section{Objetivo}

Analizar la frecuencia de experimentación y consumo de drogas en los alumnos de las escuelas de nivel medio de la provincia de Córdoba en Argentina en relación a variables sociodemográficas en el año 2005.

\section{Metodología}

Se trata de un estudio observacional, analítico y de correlación, el cual fue efectuado en base al registro realizado por la Secretaría de Programación para la Prevención de la Drogadicción y la Lucha contra el Narcotráfico (SEDRONAR) a partir de la Segunda Encuesta Nacional en Estudiantes de Enseñanza Media
2005 ( $n=4594)$, en la provincia de Córdoba y facilitado a través del Observatorio Argentino de Drogas (OAD). El envío de la base de datos desde el OAD se efectuó con un código de identificación para cada encuestado, el cual fue confidencialmente resguardado en el proceso de investigación. Los pasos en el desarrollo del proyecto fueron, en primera instancia, el diagnóstico de los datos de la base de OAD/SEDRONAR, teniendo en cuenta la categorización de las variables sociodemográficas presentadas en el Libro de Códigos de SEDRONAR. Luego se realizó el análisis de la información respecto a la frecuencia de la experimentación y consumo de los adolescentes escolarizados.

\section{Población y Muestra}

La recolección de los datos fue efectuada a partir de una muestra proababilística estratificada, polietápica, de alumnos, tomando como universo la base de escuelas del Censo Educativo 2004 de la provincia de Córdoba; se tomaron en cuenta los totales de alumnos dados por el Censo Educativo.

La estratificación de la base de datos fue por tipo de establecimiento (tipo de colegio: privado, público, y sin datos). La encuesta se realizó sobre una muestra representativa de alumnos que tenian 13, 15 y 17 años, de la provincia de Córdoba, que corresponde a los años $1^{\circ}, 3^{\circ}$ y $5^{\circ}$ del Regimen Educativo Tradicional o Nivel Medio de la provincia de Córdoba.

En Córdoba la muestra fue de 4.593, correspondiendo a 1.968 hombres y 2.625 mujeres.

\section{Variables estudiadas}

Las variables analizadas fueron: prevalencia mes (estudiantes que manifestaron haber consumido alguna sustancias en los últimos 30 días o primer contacto), prevalencia año (consumo del último año anterior a la encuesta, el cual puede ser entendido como consumo reciente), prevalencia vida (consumo realizado por los estudiantes en algún momento de la vida).

Las variables clasificatorias fueron: intervalos de edad, sexo, tipo de establecimiento, nivel educativo y características socioeconómicas.

\section{Evaluación Estadística}

El análisis descriptivo de las variables fue efectuado a partir de las medidas de resumen. El procesamiento estadístico de los datos se efectuó como análisis bivariado, a través de datos categóricos (test de chi -cuadrado, Mantel Haenzel o test de Fisher) obteniéndose la razón de riesgo y los intervalos de confianza (IC) para cada 
variable estudiada. En todos los casos se estableció un nivel de significación de $p<0,05$.

\section{Resultados}

A continuación se presenta el análisis de la frecuencia de experimentación y consumo de drogas de los alumnos de las escuelas de nivel medio de la provincia de Córdoba en relación a variables sociodemográficas. Entre las características sociodemográficas que presentaron los adolescentes escolarizados de la provincia se observó que la media de edad fue de $14,9 \pm 0,03$, con un rango entre los 11 y 22 años (Figura 1).

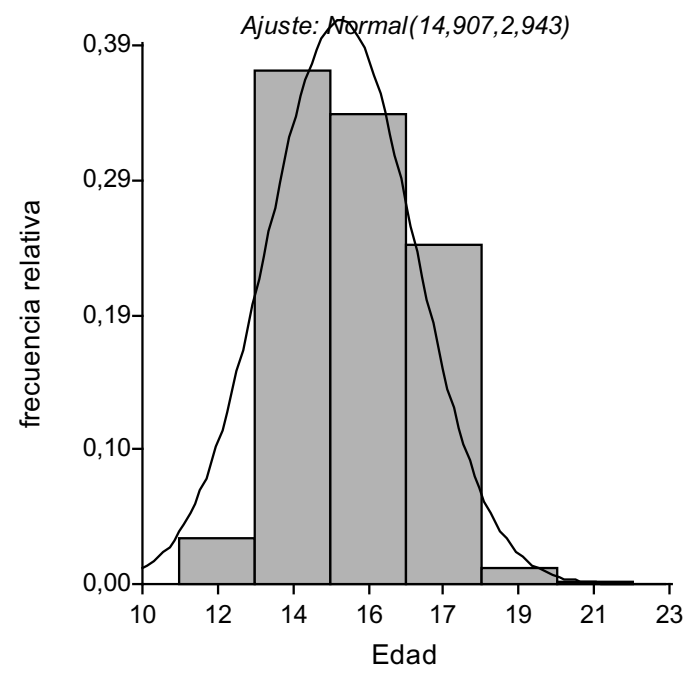

Figura 1- Media de edad de la muestra de adolescentes escolarizados de la provincia de Córdoba

El $42,8 \%$ eran alumnos de sexo masculino y $57,1 \%$ femenino. La media de edad para los alumnos del sexo masculino fue del $14,9 \pm 0,04$ años y para las mujeres del $14,8 \pm 0,03$ años.

Cuando se observó el tipo de colegio al que concurrían los adolescentes, resultó que el 54,4\% de los estudiantes escolarizados concurrían a escuelas públicas, el resto a colegios privados, y el 73, 74\% del total asistía al turno de la mañana.

Respecto al año de cursado de la escuela de nivel medio, el 38,4\% concurría al $1^{a}$ año, el 32,9\% al tercer año, y el $28,6 \%$ concurría al quinto año del Regimen Educativo Tradicional o Nivel Medio.

Cuando relacionamos las variables edad y tipo de colegio se observó una media de edad mayor para colegios públicos (14,9 años) y para los colegios privados 14,8 años $(p<0,01)$.

Respecto a las características de las familias de los adolescentes escolarizados se detectó que el 69,9\% de los padres estaban casados, 5,2\% divorciados, $12 \%$ separados, $6,6 \%$ unidos en convivencia, $2,4 \%$ solteros, $3,8 \%$ viudos y el nivel de no respuesta, de los estudiantes, fue de $1,4 \%$. En cuanto a la convivencia manifestaron, en el $75,5 \%$ de los casos, que viven con el padre y la madre, el 0,9\% con el padre y su pareja, el $4,6 \%$ madre y su pareja, el $2,2 \%$ sólo con el padre, el $13,6 \%$ sólo con la madre y el $2,2 \%$ con ninguno de los dos.

Respecto a las condiciones socioeconómicas de las familias de los estudiantes, fue encontrado que el $55,3 \%$ de los estudiantes pensaban que la condición socioeconómica de su familia era buena, 29,6\% creían que la condición era regular, 3,3\% que era mala y $4,8 \%$ no sabía.

El 61,6\% de los adolescentes escolarizados contaban con hasta 60 pesos de disponibilidad de dinero mensual y el $37,3 \%$ con más de 60 pesos. Cuando los adolescentes procedían de colegios públicos el $67,6 \%$ contaban con hasta 60 pesos y en los privados fueron el $61,7 \%$.

A partir del análisis descriptivo de la base de datos (Figura 2), a respecto a la prevalencia de vida (consumo realizado por los estudiantes en algún momento de la vida), el alcohol y el tabaco son las sustancias de mayor consumo. Asimismo, el $12,2 \%$ de los estudiantes de la provincia de Córdoba probó alguna vez en la vida alguna droga ilícita. Dentro del conjunto de psicofármacos usados sin prescripción médica, los tranquilizantes ocupaban el primer lugar. 


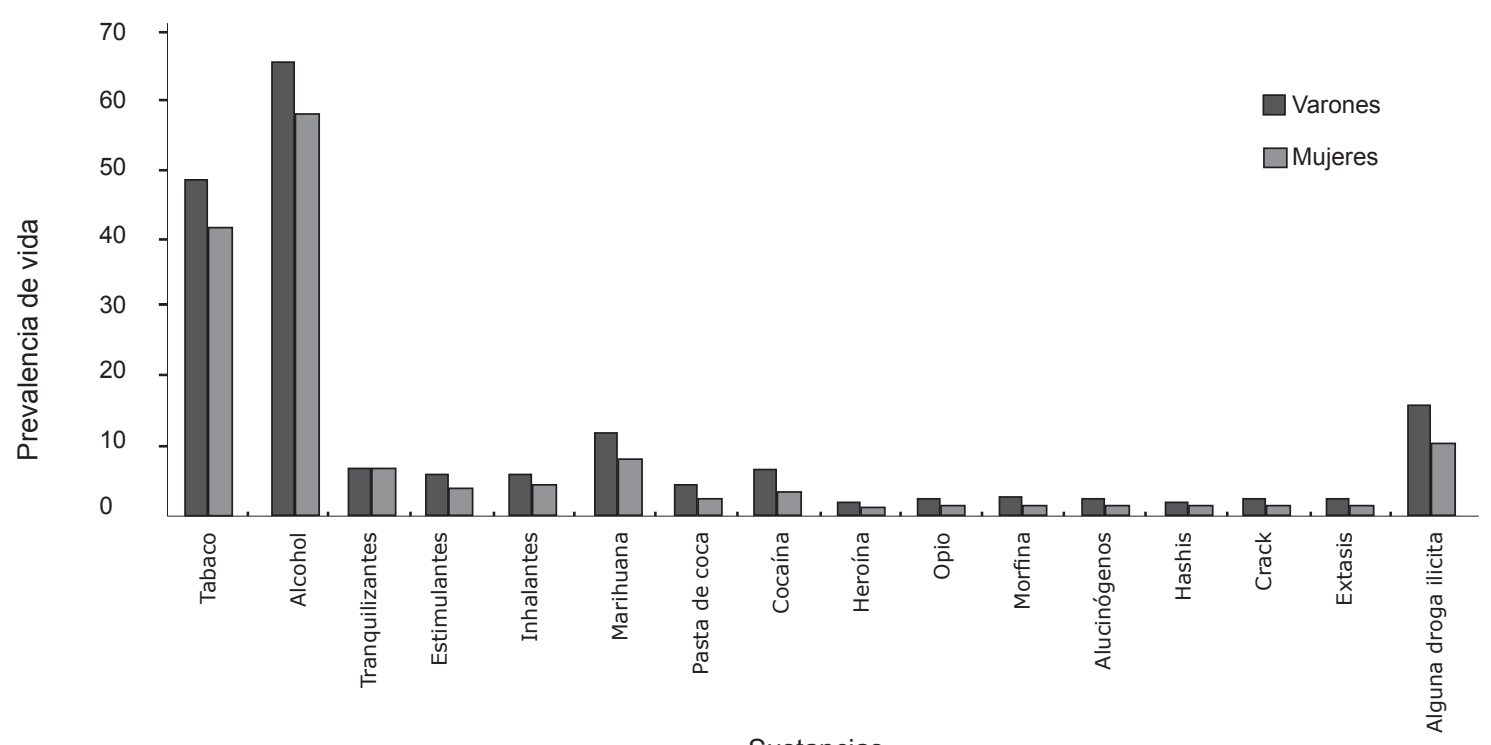

Sustancias

Figura 2- Prevalencia en la vida por tipo de sustancias psicoactivas según sexo en la muestra de la población escolar de nivel medio de la provincia de Córdoba en el año 2005

Respecto a cualquier consumo realizado en el año anterior a la encuesta (o prevalencia año) se observó que el $52,8 \%$ de los hombres consumieron alcohol, siendo esta una prevalencia mayor que en las mujeres (45,6\%, $\mathrm{p}<0,0001)$. El 48,9\% de los adolescentes escolarizados consumieron alguna bebida alcohólica durante el último año; si se tiene en cuenta a los adolescentes que no contestaron la pregunta, se puede destacar que esta frecuencia se incrementa al $87 \%$ cuando sólo tenemos en cuenta los estudiantes que sí respondieron a ésta pregunta $(56,2 \%)$. El odd ratio (OR) respecto al consumo de alcohol anual mostró un riesgo dos veces mayor en la provincia de Córdoba que en el promedio del país, con la Ciudad Autónoma de Buenos Aires, y con provincias como Buenos Aires, Santa Fe, y Jujuy.

Cuando la variable analizada es la prevalencia de consumo de tabaco en el último año, no se observaron diferencias según el género, siendo $26 \%$ para hombres y $26,8 \%$ para mujeres. Respecto a drogas ilícitas, el $7 \%$ de los estudiantes presentan consumo reciente.

Teniendo en cuenta que la prevalencia de mes corresponde a los estudiantes que manifestaron haber consumido alguna sustancia en los últimos 30 días, también se consideró a este consumo como primer contacto, y se observó que el 22,5\% de los estudiantes fumaron y el $44 \%$ ingirió alguna bebida alcohólica.

La prevalencia de consumo de vida, año y mes por tipo de sustancia psicoactiva según edad tiene un comportamiento similar al encontrado cuando se analiza según año de estudio. En todas las sustancias el valor de la prevalencia aumenta (tanto para vida, año y mes) a medida que aumenta el rango de edad y/o el año de estudio.

En cuanto a la edad de inicio de consumo de tabaco, el $13 \%$ manifestó iniciar el consumo a los 13 años; las menores prevalencia de inicio se encontraron en las edades 12 a 14 años. Estos resultados corresponden al 42,5\% de los que contestaron la pregunta. En cuanto al inicio del consumo de alcohol la edad más frecuente fue de 14 años, y con menor frecuencia fueron los 13, 12 y 15 años.

El primer contacto o consumo de tabaco en los últimos 30 días aumenta al incrementarse la disponibilidad de dinero con el que cuentan los adolescentes escolarizados mensualmente. Al analizar estas variables (consumo de tabaco de los estudiantes en los últimos 30 días con disponibilidad de dinero mensual) se observó que la prevalencia para los que cuentan hasta con 60 pesos es de $75,7 \%$ y de $80,3 \%$ para los que tienen más de 60 pesos mensuales $(p<0,03)$. Al efectuar el análisis de riesgo para esta frecuencia se observó que los que poseen más de 60 pesos, el riesgo de consumir tabaco en la adolescencia se incrementa en una vez. Cuando se realiza el mismo análisis respecto al consumo de alcohol de los últimos 30 días (prevalencia mes de alcohol) se observa que el $90,7 \%$ de los estudiantes que cuentan con más de 60 pesos consumieron alcohol en los últimos 30 días y el $84,8 \%$ de los que tienen hasta 60 pesos $(\mathrm{p}<$ 0,001 ) con OR de 1,75 con IC: $1,35-2,25$. Es decir que para los adolescentes que cuentan con más de 60 pesos mensuales, hay en la provincia de Córdoba, dos veces más riesgo para consumo de alcohol y una vez para el consumo de tabaco. 
Cuando analizamos esta variable con tipo de colegio, se observó que el comportamiento no se modificó según colegio público o privado. Los alumnos de colegio público que cuentan con más dinero tienen mayor consumo en el último mes para alcohol que los que tienen disponibilidad menor a 60 pesos mensuales. No se observa diferencia según disponibilidad de dinero para el consumo de marihuana pero si para cocaína que presenta un mayor riesgo $(p<0,02)$. Es de destacar que la tasa de respuesta de estas preguntas es baja, con un $5,9 \%$ para prevalencia de mes para marihuana y $2,5 \%$ para prevalencia de mes de cocaína.

\section{Discusión}

Los resultados obtenidos en relación con la prevalencia de vida de consumos de sustancia psicoactivas en adolescentes escolarizados, de la provincia de Córdoba, coinciden con los observados en el análisis del ámbito nacional(11). El alcohol y el tabaco son las sustancias de mayor consumo, según el análisis de prevalencia de vida de este trabajo. El 48,7\% de los adolescentes encuestados consumió alguna bebida alcohólica, valores cercanos al nacional, el cual corresponde al $48 \%$. Otros estudios demuestran que el alcohol es la droga más utilizada entre adolescentes escolarizados en el mundo(12-17). En los adolescentes escolarizados, de la provincia de Córdoba, se observa que en el consumo de tabaco no hay diferencias entre hombres y mujeres $y$ en alcohol son los hombres los que tienen la mayor tasa de consumo. En ambos casos se repite el patrón de consumo a nivel nacional. En un estudio en el año 2001, en Brasil, se muestra un comportamiento similar de consumo de alcohol y tabaco, con mayor consumo de alcohol en hombres y tendencia al equilibrio en el consumo de tabaco, esto de acuerdo al género(18). Al igual que en otras ciudades de Latinoamérica el consumo de drogas ilícitas en mujeres es de menor proporción que en hombres ${ }^{(5,19)}$.

El $7 \%$ de los estudiantes, de la provincia en estudio, presenta valores de consumo reciente de alguna droga ilícita que se asemejan a la media nacional(7,1\%).

En todas las sustancias la prevalencia aumenta (tanto para vida, año y mes) a medida que aumenta el rango de edad y/o el año de estudio, con excepción de estimulantes, inhalantes y pasta de coca.

En la prevalencia de consumo de drogas ilícitas, la marihuana ocupa el primer lugar, seguido por tranquilizantes, inhalantes y en último lugar con 3,9\% se encuentran los estimulantes y la cocaína. Esto coincide parcialmente con lo observado en estudios realizados en
Brasil en donde la marihuana es la droga ilícita con mayor prevalencia, pero seguida por los inhalantes ${ }^{(18,20-21)}$.

Cuando relacionamos la frecuencia de experimentación y consumo de drogas de los alumnos de la escuela media de la provincia de Córdoba con las variables sociodemográficas, se observa que el consumo de alcohol y drogas ilegales es más frecuente en el sexo masculino y en los que tienen mayor disponibilidad de dinero. No se observó diferencia entre los adolescentes que asisten a escuelas públicas y privadas. Pero se sabe que la asistencia a la escuela es un factor protector importante para el consumo(5).

Este estudio reveló que el primer contacto o consumo de tabaco en los últimos 30 días aumenta al incrementarse la disponibilidad de dinero con el que cuentan los adolescentes escolarizados mensualmente, esto está de acuerdo con un estudio que describe que el uso de las sustancias ilícitas es mayor en la burguesía(18). La asociación entre disponibilidad de dinero y uso de drogas se demostró en varios estudios en otros países del mundo(22-24).

\section{Conclusión}

El presente estudio detectó las características que presentaban con mayor frecuencia y describían fidedignamente a los adolescentes escolarizados que experimentaron $\mathrm{y} / \mathrm{o}$ consumieron drogas, también identificó a los grupos más vulnerables. Conocer estos datos permite definir estrategias de prevención acordes con las necesidades de este grupo poblacional. Es importante destacar que esta investigación refleja los resultados del análisis de una muestra de adolescentes escolarizados y que sus resultados no pueden extrapolarse para la población general de adolescentes.

\section{Agradecimientos}

Agradecemos a la Comisión Interamericana para el Control del Abuso de Drogas/CICAD de la Secretaria de Seguridad Multidimensional/SSM de la Organización de los Estados Americanos/OEA, la Secretaria Nacional de Políticas sobre Drogas/SENAD do Gabinete de Seguridad Institucional/Brasil, la Escuela de Enfermería de Ribeirao Preto de la Universidad de Sao Paulo y Centro Colaborador de la Organización Mundial de la Salud para el Desarrollo de la Investigación en Enfermería, la población representada en los estudios de investigación, bien como a las autoridades de las universidades representadas por los participantes del Programa En-Line de Especialización en Investigación sobre el Fenómeno de las Drogas PREINVEST, periodos 2005, 2006, 2007 y 2008. 


\section{Referencias}

1. Robertson E, David S, Rao S. ¿Cómo Prevenir el Uso de Drogas en los niños y adolescentes? Una guía con base científica para padres, educadores y líderes de la comunidad Instituto Nacional sobre el Abuso de Drogas. NIDA. 2 ed. (versión abreviada) Maryland (USA): NIDA; 2004.

2. Pentz MA. Costs, benefits, and cost effectiveness of comprehensive drug abuse prevention. In: Bukoski WJ. Cost Effectiveness and Cost Benefit Research of Drug Abuse Prevention: Implications for Programming and Policy. Monografía de investigación del NIDA. Maryland (USA): NIDA; 2003.

3. Fernández $S$, Nebot $M$, Jané M. Evaluación de la efectividad de los programas escolares de prevención del consumo de tabaco, alcohol y cannabis: ¿Qué nos dicen los meta-análisis? Rev Esp Salud Pública. 2002;76:175-87.

4. Hawkins JD, Catalano RF, Arthur M. Promoting science-based prevention in communities. Addic Behav. 2002;90(5):1-26.

5. Medina-Mora ME, Craviota P, Villatoro J, Fleiz C. Consumo de drogas entre adolescentes: resultado de la encuesta nacional de adicciones, 1998. Salud Pública Méx. 2003;45 supl 1:16-25.

6. Secretaría de Programación para la Prevención de la Drogadicción y la Lucha contra el Narcotráfico - SEDRONAR (AR). Resultado de la primer Encuesta Nacional a Estudiantes de la Enseñanza Media. Argentina: SEDRONAR; 2001.

7. Fraguela JAG, Martín AL, Triñanes ER. Prevención del consumo de drogas en la escuela: cuatro años de seguimiento de un programa. Psycothema. 2002; 14 (4): 685-92.

8. Tobler N, Stratton H. Effectiveness of School-Based drug Prevention Programs: A Meta-Analysis of the Research. J Primary Prev. 1997; 18 (1):71-128.

9. Tobler N, Roona M, Oschshorns P, Marshall D, Streke A, Stakpole K. School based adolescent drug prevention programs: 1998 Meta-analysis. Prim Prevent. 2000; 20(4):275-336.

10. Wilson D, Grottfredson D, Najaka S. School-based prevention of problem behaviors: A meta-analysis. Quant Criminol. 2001;17(3):247-72.

11. Secretaría de Programación para la Prevención de la Drogadicción y la Lucha contra el Narcotráfico - SEDRONAR (AR). Informe Final de la Encuesta Nacional a Estudiantes de la Enseñanza Media. Argentina: SEDRONAR; 2005.

12. Matute, RC, Pillon, SC. Alcohol consumption by nursing students in Honduras. Rev. Latino-Am. Enfermagem. 2008;16(spe):584-9.
13. Patton GC, Hibbert M, Rosier MJ, Carlin JB, Caust J, Bowes G. Patterns of common drug use in teenagers. Austr Public Health. 1995; 19:393-9.

14. Pecci MC. Varones y Jóvenes Sustancias Psicoactivas. Acta Psiquiátr Psicol Am Lat. 1995;41:288-99.

15. Barroso T, Mendes A, Barbosa A. Análise do fenômeno do consumo de álcool em adolescentes: estudo realizado com adolescentes do $3^{\circ}$ ciclo de escolas públicas. Rev. Latino-Am. Enfermagem. 2009;17(3):347-53.

16. Galduroz JCF, Noto AR, Carlini EA. IV Levantamento sobre o uso de drogas entre estudantes de $1^{\circ}$ e $2^{\circ}$ graus em 10 capitais brasileiras. São Paulo: CEBRID Centro Brasileiro de Informações sobre drogas psicotrópicas. Universidade Federal Paulista: Departamento de Psicologia, Escola Paulista de Medicina; $1997.130 \mathrm{p}$.

17. Muza GM, Bettiol H, Muccillo G, Barbieri MA. Consumo de substancias psicoativas por adolescentes escolares de Ribeirão Preto, SP (Brasil). Rev Saúde Publica. 1997;31:163-70.

18. Tavares BF, Béria JU, Lima MS. Prevalência do use de drogas e desempenho escolar entre adolescentes. Rev Saúde Publica. 2001;35(2):150-8.

19. Jinez LJ, Souza JRM, Pillon Sandra Cristina. Uso de drogas e fatores de risco entre estudantes de ensino médio. Rev. LatinoAm. Enfermagem. 2009;17(2):246-52.

20. Villatoro JA, Medina-Mora ME, Cardiel H, Fleiz CM, Alcantares E, Hernandes S. La situación del consumo de sustancias entre estudiantes de la ciudad de México: medición otoño 1997. Salud Mental. 1999;22:18-30.

21. Tapia-Conyer R, Kuri P, Cravioto P, Revuelta A. Sistema de Vigilancia Epidemiológica de las Adicciones (SISVEA). Informe. México (DF): Secretaría de Salud; 1998.

22. Beutelspacher NA, Conyer RT, Romero AV, Alvarez GL, Mora MEM, Izaba BS. Factores asociados al consumo de drogas en adolescentes de áreas urbanas de México. Salud Pública Méx. 1994;36:646-54.

23. Cardenal CA, Adell MN. Consumo de alcohol en escolares. Med Clín. 1995;105:481-6.

24. Singh H, Mustapha N. Some factors associate with substance abuse among secondary school students in Trinidad and Tobago. Drug Educ. 1994;24:83-93.

Recibido: 24.2.2010

Aceptado: 5.5.2010 